\title{
Analytical solution for the side-fringing fields of narrow beveled heads
}

\author{
I. D. Mayergoyz, R. Madabhushi, E. R. Burke, a) and R. D. Gomez \\ Department of Electrical Engineering, University of Maryland, College Park, Maryland, 20742
}

By using conical coordinates, exact analytical solutions for three-dimensional side-fringing fields of recording heads that are beveled in the down-track direction are found. These solutions are derived under the assumption of zero gap length. The side-fringing fields for the two limiting cases of infinitesimally narrow heads and semi-infinitely wide heads are presented and compared. (C) 1997 American Institute of Physics. [S0021-8979(97)29508-3]

\section{INTRODUCTION}

Recently, the focused ion beam micromachining (FIBM) technique has been introduced for the patterning of the pole tips of recording heads. This technique has already demonstrated the ability to achieve very narrow head geometries, and it is expected to be very instrumental in the patterning of various pole tip configurations. ${ }^{1,2}$ This suggests the importance of the analysis of side-fringing fields of very narrow heads. In this article, exact analytical solutions for the threedimensional side-fringing fields are presented. First, the case of infinitesimally narrow heads beveled in the down-track direction is discussed under the assumption of zero gap length. It is apparent that the side fringing fields of finite width heads may deviate from those which are found for infinitesimally narrow heads. To estimate the range of these deviations, exact analytical solutions for the threedimensional fringing fields of semi-infinitely wide heads are then derived. Finally, differences in the strength and the cross track extent of the side-fringing fields for these two head geometries are analyzed for various bevel angles.

\section{INFINITESIMALLY NARROW HEADS}

The idealized geometry of such heads is shown in Fig. 1(A). It is assumed that: (a) the pole tips are semi-infinite in extent in the $x-y$ plane, (b) the gap length is equal to zero, and (c) the magnetic scalar potential $\Psi$ has constant values of $\Psi_{0}$ and $-\Psi_{0}$ on opposite sides of the gap. The specific value of $\Psi_{0}$ is determined by the ampere turns and the efficiency of the recording head.

It is apparent that the potential $\Psi$ satisfies the Laplace equation

$$
\nabla^{2} \Psi=0
$$

in the region outside the magnetic head. This potential is also subject to the boundary conditions:

$$
\begin{aligned}
& \Psi=\Psi_{0} \quad \text { for } \theta=\frac{\pi}{2}, \quad \frac{3 \pi}{2}<\varphi \leqslant \frac{5 \pi}{2}-\gamma, \\
& \Psi=-\Psi_{0} \quad \text { for } \theta=\frac{\pi}{2}, \quad \gamma+\frac{\pi}{2} \leqslant \varphi<\frac{3 \pi}{2},
\end{aligned}
$$

where $\theta$ and $\phi$ are spherical coordinates, while $\gamma$ is the bevel angle $(\pi / 2 \leqslant \gamma<\pi)$.

${ }^{a}$ Laboratory for Physical Sciences, College Park, MD 20740.
It is clear that the solution of the boundary value problem (1)-(3) does not depend on $r: \partial \Psi / \partial r \equiv 0$. This is because the boundary conditions (2) and (3) do not depend on $r$. In other words, there is no dimension in the above problem against which $r$ can be scaled. The above fact can be exploited in order to reduce the 3D boundary value problem (1) -(3) to the 2D boundary value problem for the Laplace equation in the region shown in Fig. 1(B). This reduction is achieved by using the conical coordinates:

$$
\alpha_{1}=\frac{-z}{r+y}, \quad \alpha_{2}=\frac{-x}{r+y}, \quad \alpha_{3}=r
$$

and the mathematical details of such a transformation can be found in previous publications. ${ }^{3,4}$ Thus, in terms of conical coordinates, $\alpha_{1}$ and $\alpha_{2}$, the boundary value problem (1)-(3) can be restated as follows: find the solution to the Laplace equation

$$
\frac{\partial^{2} \Psi}{\partial \alpha_{1}^{2}}+\frac{\partial^{2} \Psi}{\partial \alpha_{2}^{2}}=0
$$

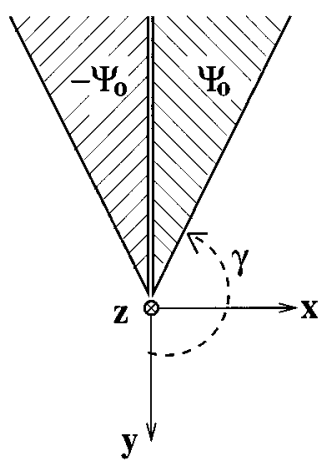

(A)

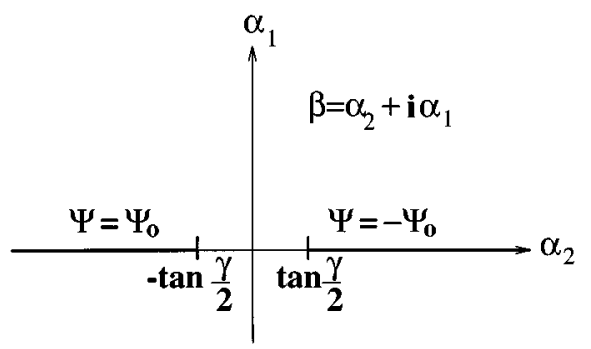

(B)

FIG. 1. (A) The idealized geometry of the infinitesimally narrow head. (B) Its conical-coordinate map. 
subject to the boundary conditions

$$
\begin{aligned}
& \Psi=\Psi_{0} \quad \text { for } \alpha_{1}=0, \quad-\infty<\alpha_{2}<-\tan \frac{\gamma}{2}, \\
& \Psi=-\Psi_{0} \quad \text { for } \alpha_{1}=0, \quad \tan \frac{\gamma}{2}<\alpha_{2}<\infty
\end{aligned}
$$

The theory of functions of complex variables can now be used to solve the boundary value problem (5)-(7). To this end, we introduce the complex variable $\beta=\alpha_{2}+i \alpha_{1}$, $i=\sqrt{-1}$, and consider the function:

$$
\Psi(\beta)=\frac{2 \Psi_{0}}{\pi} \arg \left[\beta+\sqrt{\beta^{2}-\tan ^{2} \frac{\gamma}{2}}\right]-\Psi_{0} .
$$

This function is the imaginary part of the analytical function $W(\beta)=\left(2 \Psi_{0} / \pi\right) \ln \left[\beta+\sqrt{\beta^{2}-\tan ^{2}(\gamma / 2)}\right]-i \Psi_{0}$, and, for this reason, it satisfies the Laplace Eq. (5). It is also easy to check that $\Psi(\beta)$ given by (8) satisfies the boundary conditions (6) and (7). Thus, it is established that expression (8) is indeed the solution for the boundary value problem (5)-(7). By using formulas (8) and (4) and straightforward (but somewhat lengthy) transformations, the following explicit expressions can be derived for the magnetic field components $H_{x}, H_{y}$, and $H_{z}$ in terms of Cartesian coordinates:

$$
\begin{aligned}
& H_{x}=\Psi_{0} \frac{\sqrt{2}\left[2 y r-\left(y^{2}+z^{2}+y r\right)\left(A+1+\tan ^{2} \frac{\gamma}{2}\right)\right]}{\pi A B r(r+y)^{2}}, \\
& H_{y}=\Psi_{0} \frac{\sqrt{2} x\left(A-\frac{x^{2}+z^{2}}{(r+y)^{2}}+\tan ^{2} \frac{\gamma}{2}\right)}{\pi A B r(r+y)}, \\
& H_{z}=\Psi_{0} \frac{\sqrt{2} x z\left(A+1+\tan ^{2} \frac{\gamma}{2}\right)}{\pi A B r(r+y)^{2}}
\end{aligned}
$$

where

$$
\begin{aligned}
& A=\left\{\left[\frac{x^{2}+z^{2}}{(r+y)^{2}}-\tan ^{2} \frac{\gamma}{2}\right]^{2}+\frac{4 x^{2} z^{2}}{(r+y)^{4}}\right\}^{1 / 2}, \\
& B=\left[A+\frac{z^{2}-x^{2}}{(r+y)^{2}}+\tan ^{2} \frac{\gamma}{2}\right]^{1 / 2} .
\end{aligned}
$$

\section{SEMI-INFINITELY WIDE HEADS}

The idealized geometry of such heads is shown in Fig. 2(A). It is assumed that: (a) the pole tips are semi-infinite in extent in the $z$ direction as well as in the $x-y$ plane, (b) the gap length is equal to zero, and (c) the magnetic scalar potential has constant values of $\Psi_{0}$ and $-\Psi_{0}$ on opposite sides of the gap. This leads to the boundary value problem of finding the solution to the Laplace equation

$$
\nabla^{2} \Psi=0,
$$

subject to the boundary conditions

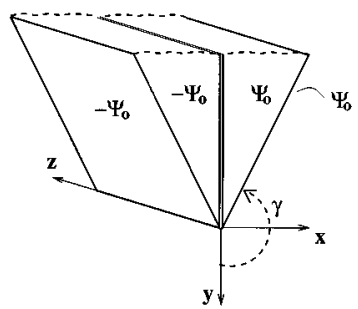

(A)

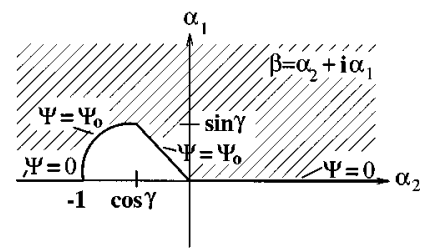

(B)

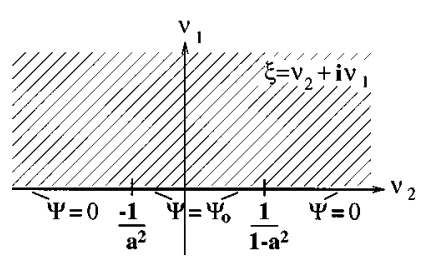

(C)

FIG. 2. (A) The idealized geometry of the semi-infinitely wide head. (B) Its conical-coordinate map. (C) The resulting conformal map. Shaded regions map volume external to head.

$$
\Psi=-\Psi_{0} \quad \text { for } \varphi=\frac{\pi}{2}+\gamma, \quad 0 \leqslant \theta \leqslant \frac{\pi}{2}
$$

and

$$
\begin{aligned}
& \frac{\pi}{2}+\gamma \leqslant \varphi<\frac{3 \pi}{2}, \quad \theta=\frac{\pi}{2}, \\
& \Psi=\Psi_{0} \quad \text { for } \varphi=\frac{5 \pi}{2}-\gamma, \quad 0 \leqslant \theta \leqslant \frac{\pi}{2}
\end{aligned}
$$

and

$$
\frac{3 \pi}{2}<\varphi \leqslant \frac{5 \pi}{2}-\gamma, \quad \theta=\frac{\pi}{2} .
$$

Again, it is apparent that the solution of the boundary value problem (14)-(16) does not depend on $r: \partial \Psi / \partial r \equiv 0$. This suggests that an approach similar to that for the infinitesimally narrow head can be taken to solve the above boundary value problem.

As before, by using conical coordinates:

$$
\alpha_{1}=\frac{x}{r+z}, \quad \alpha_{2}=\frac{y}{r+z}, \quad \alpha_{3}=r,
$$

and the symmetry with respect to the plane $x=0$, the above $3 \mathrm{D}$ boundary value problem can be reduced to the $2 \mathrm{D}$ boundary value problem of finding the solution to the Laplace equation

$$
\frac{\partial^{2} \Psi}{\partial \alpha_{1}^{2}}+\frac{\partial^{2} \Psi}{\partial \alpha_{2}^{2}}=0
$$




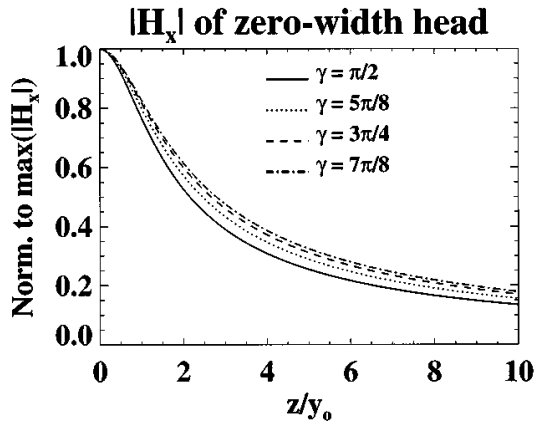

FIG. 3. $\left|H_{x}\right|$ of the infinitesimally narrow head decaying over the crosstrack direction $z$, for various bevel angles $\gamma$, at $y_{0}=0.1 \mu \mathrm{m}$.

in the shaded region of Fig. 2(B) subject to the boundary conditions indicated in the same figure.

To solve the latter boundary value problem, we introduce complex variables:

$$
\beta=\alpha_{2}+i \alpha_{1}, \quad \xi=\nu_{2}+i \nu_{1} .
$$

It can be shown ${ }^{5}$ that the function $\beta(\xi)$ defined by the expressions

$$
\beta=\frac{1+\alpha \lambda}{1-a \lambda}\left(\frac{\lambda-1}{\lambda+1}\right)^{a}, \quad \lambda=\sqrt{\frac{\xi}{1+a^{2} \xi}}, \quad a=\frac{\gamma}{\pi},
$$

conformally maps the upper half-plane shown in Fig. 2(C) into the upper half-plane with a sector bulge shown in Fig. 2(B). The boundary conditions for $\Psi(\xi)$ are indicated in Fig. 2(C).

By invoking the same line of reasoning as before, it can be demonstrated that $\Psi(\xi)$ is given by the expression

$$
\Psi(\xi)=\frac{\Psi_{0}}{\pi} \arg \left(\frac{\xi-\frac{1}{1-a^{2}}}{\xi+\frac{1}{a^{2}}}\right) .
$$

By using the last expression and coordinate transformations (17), (19), and (20), the magnetic scalar potential and magnetic field can be computed in terms of Cartesian coordinates. For the sake of brevity, mathematical details of these calculations are omitted.

\section{NUMERICAL RESULTS}

Using the analytical solutions for the magnetic fields derived above for the two head geometries, the writing characteristics of the narrow beveled head have been studied. The down-track magnetic field components, $H_{x}$, have been computed and compared for different bevel angles. First, for the zero-width head, the decay of $H_{x}$ in the cross-track $(\hat{z})$ direction was considered as a function of bevel angle. Then, $H_{x}$ as a function of the down-track $(\hat{x})$ direction was computed for several bevel angles in order to study the field gradient in the down-track direction. And finally, the decay of $H_{x}$ in the $\hat{z}$ direction was compared with that of the semiinfinite head width geometry.

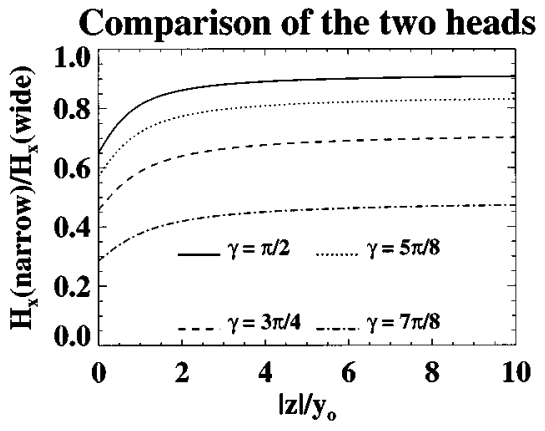

FIG. 4. $H_{x}$ (zero width) $/ H_{x}$ (semi-infinite width) decaying over $z$ for various $\gamma$, at $y_{0}=0.1 \mu \mathrm{m}$.

Figure 3 presents $H_{x}$ created by the zero-width head as a function of $z$, for various values of the bevel angle $\gamma$. Here $H_{x}$ is shown to decay somewhat slower over $z$ as the bevel angle increases.

Since computations also show that $H_{z}$ decays slower in $z$ as $\gamma$ increases for the infinitesimally narrow head, the question arises as to the purpose of beveling. Computations show that $H_{x}$ drops off faster in $x$ as $\gamma$ increases, where $\Psi_{0}$ is scaled appropriately to allow the maximum of $H_{x}$ to be independent of $\gamma$. This increase in field gradient allows narrower transitions to be written in the recording medium, thereby increasing the down-track density. Therefore, the fact that the magnetic fields decrease slower with $\gamma$ in the cross-track direction and decay faster with $\gamma$ in the downtrack direction is an issue that must be deliberated when beveling is considered.

Although beveling is a foreseeable application of FIBM, decreasing the width of thin-film heads has been the main use of this technique in the patterning of these heads. Therefore, another issue is the head width dependence of the fringing fields. In Fig. 4, the ratio of $H_{x}$ created by the zero-width head to $H_{x}$ created by the semi-infinitely wide head varying over $z$ is displayed. The magnetic field ratio decreases as a function of bevel angle; for $z=0.5 \mu \mathrm{m}$, it varies from a relative difference of about $10 \%$ at $\gamma=\pi / 2$ to a difference of about 55\% at $\gamma=7 \pi / 8$. For a head of finite width, the curves would lie somewhere between unity and the curves of the figure. Also, by showing that the aforementioned ratio increases away from the side of the head, the figure reveals that the semi-infinitely wide head creates fields that decay more rapidly in $z$ than the fields created by the zero-width head. This fact must be taken into account when considering the increase of track density.

${ }^{1}$ C. Tsang, H. Santini, D. McCown, J. Lo, and R. Lee, IEEE Trans. Magn. 32, 7 (1996).

${ }^{2}$ W. P. Jayasekara, S. Wang, and M. H. Kryder, J. Appl. Phys. 79, 5880 (1996).

${ }^{3}$ I. D. Mayergoyz and D. S. Bloomberg, IEEE Trans. Magn. Mag-22, 163 (1986).

${ }^{4}$ I. D. Mayergoyz and D. S. Bloomberg, J. Appl. Phys. 63, 3381 (1988).

${ }^{5}$ V. I. Ivanov and M. K. Trubetskov, Handbook of Conformal Mapping with Computer-Aided Visualization (Chemical Rubber, Boca Raton, 1995), p. 311. 
Journal of Applied Physics is copyrighted by the American Institute of Physics (AIP). Redistribution of journal material is subject to the AIP online journal license and/or AIP copyright. For more information, see http:/ojps.aip.org/japo/japcr/jsp

Copyright of Journal of Applied Physics is the property of American Institute of Physics and its content may not be copied or emailed to multiple sites or posted to a listserv without the copyright holder's express written permission. However, users may print, download, or email articles for individual use. 